\title{
Diagnosing and sports counselling of athletes with myocarditis
}

CARDIOLOGY / SPORTS MEDICINE

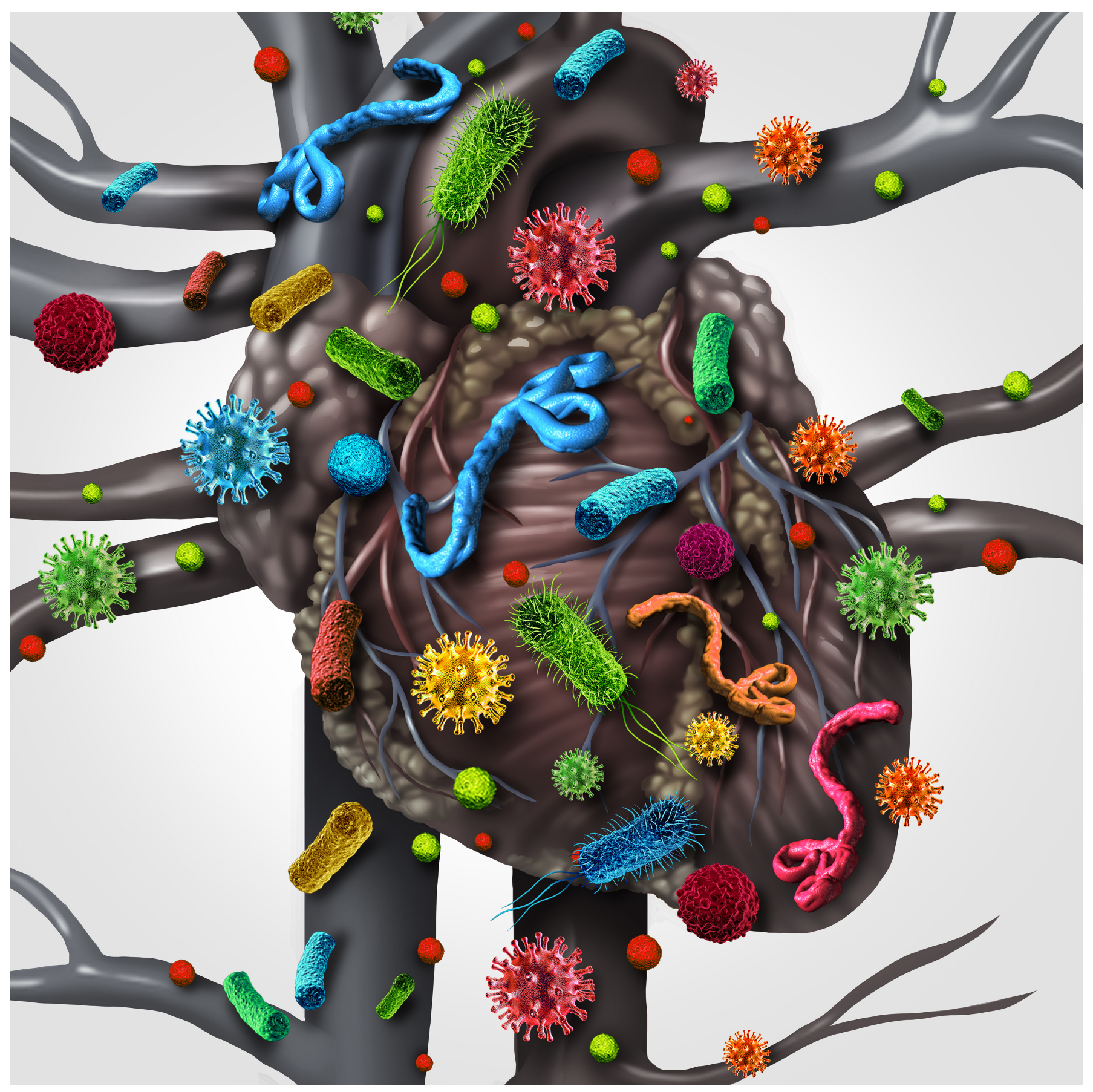




\title{
Eichhorn $\mathrm{C}^{1},{ }^{2}$, Gräni $\mathrm{C}^{1}{ }^{2}$
}

${ }^{1}$ Department of Cardiology, Swiss Cardiovascular Center, University Hospital Berne, Switzerland

${ }^{2}$ Noninvasive Cardiovascular Imaging Section, Cardiovascular Division, Department of Medicine, Brigham and Womens Hospital, Harvard Medical School, Boston, USA

\begin{abstract}
Myocarditis is defined as an inflammation of the heart muscle and its presentation, especially in athletes, is heterogeneous. Underlying causes include in most of the cases viruses, and less often bacteria, toxins, vasculitic diseases or pharmaceutical agents. Cardiac magnetic resonance (CMR) imaging is the primary imaging tool to diagnose myocarditis following laboratory test, electrocardiogram and echocardiography. In certain cases, endomyocardial biopsy is required, especially in unclear cases with reduced systolic left ventricular ejection fraction. Although, athletes and sport physicians face the dilemma of significant performance decline in competitive athletes against the risk of adverse cardiac events, currently abstinence from competitive sports is recommended for at least 3-6 months in myocarditis. Sports recommendations are currently based mainly on autopsy studies and experts' opinions and better risk stratification tools are imperatively needed. New tissue characterization methods, namely T1 mapping and T2 mapping in CMR continue to improve sensitivity and specificity of diagnosing myocarditis and may further enhance individual risk assessment. In the future, sports physicians may be able to rely more on these novel noninvasive tissue characterisation methods in risk stratification and sports restriction recommendations of athletes with suspected myocarditis.
\end{abstract}

\section{Zusammenfassung}

Myokarditis ist eine Herzmuskelentzündung mit heterogener klinischer Präsentation, dies insbesondere bei Athleten. Die häufigsten Ursachen umfassen Virusinfektionen, oder weniger häufig bakterielle Infektionen, Vaskulitis, Toxine oder Medikamente. Neben den laborchemischen Untersuchungen, dem Elektrokardiogramm und der Echokardiographie, ist die kardiovaskuläre Magnet-Resonanz-Tomographie (MRT) aktuell die primäre Untersuchungsmethode in diesem klinischen Zusammenhang. Bei gewissen Patienten mit Verdacht auf Myokarditis, insbesondere wenn sich eine unklar reduzierte systolische Ejektionsfraktion zeigt, ist die endomyokardiale Biopsie ein weiteres wichtiges Diagnoseinstrument. Aktuell ist bei Athleten mit einer Myokarditis mindestens 3-6 Monate kompetitive Trainingspause empfohlen, was der Wettbewerbsfähigkeit eines Athleten nachhaltig schaden kann. Diese Empfehlungen sind hauptsächlich basierend auf Autopsiestudien und Expertenmeinungen entstanden. Neue Gewebecharakterisierungs-Methoden im kardiovaskulären MRT, namentlich T1 mapping und T2 mapping, verbessern die Sensitivität und Spezifizität der Myokarditis-Diagnostik. Zukünftig werden Sportmediziner ihre Athleten mit Verdacht auf eine Myokarditis besser risikostratifizieren können und bezüglich Sportverhaltens je nach Befund der nichtinvasiven kardialen Bildgebung besser beraten können. 


\section{Introduction}

Myocarditis, defined as inflammation of the myocardium, presents the third most common cause of sudden cardiac death (SCD) in young athletes after autopsy-negative sudden unexplained death and coronary artery anomalies in the USA and Italy [1,2]. Also in post-mortem studies of athletes in Switzerland, we could recently show that myocarditis was one of the underlying causes of SCD [3,4]. However, whether exercise is a trigger of fatal arrhythmias is an unanswered question as the majority of SCD occurs at rest and not during or immediately after physical activity [1]. While exercise is undeniably beneficial for cardiovascular health [5], excessive exercise has been shown to predispose athletes to infection and exacerbate it [2]. Commonly, myocarditis is caused by viral infection such as enterovirus, Coxsackie B, Parvovirus B19 and Human Herpes Virus 6 [6,7]. Non-infectious causes, which are generally rare, include toxins, vasculitic diseases or pharmaceutical agents and illicit drugs [8]. The question amongst primary care physicians, sports physicians and cardiologists alike is how we can best identify athletes who may be suffering from myocarditis, which inevitably predisposes them to a higher risk of SCD, and how we risk stratify these athletes. Currently, based on animal and autopsy studies, expert opinions recommend an abstinence from competitive sports for 3 to 6 months after an episode of myocarditis

[9-12]. This, however, can be of great inconvenience to athletes due to deconditioning and an inability to compete. This review aims to present the current challenges in diagnosing myocarditis and further propose how to approach this clinical setting using Cardiac magnetic resonance (CMR) imaging (see Figure 1). The presented flow-chart incorporates clinical, laboratory and imaging markers with the aim to better diagnose and risk stratify athletes with suspected myocarditis.

\section{Contributing effect of exercise on pathogenesis}

Intense endurance training, compared to moderate exercise, which is generally protective, can predispose to viral upper respiratory tract infection and increases the chance of a systemic inflammatory response [13]. Murine studies have shown that this inflammatory response in athletes is associated with a higher propensity to arrhythmogenicity [14]. In addition to that, in athletes affected by myocarditis, infectious agents attack the myocardium directly and cause a subsequent autoimmune response that leads to myocardial remodelling through three phases: acute viral, subacute immune and chronic phase, where severe cases develop into a dilated cardiomyopathy [15].

In summary, the interaction between an environmental trigger and the host's immune system creates a subacute and chronic inflammatory state. This leads to death of myocardial tissue and causes scarring of the heart, which further predisposes individuals to arrhythmias. Even beyond an episode of myocarditis, it is believed that chronic myocardial inflammation secondary to pathogenic autoimmunity can persist due to the continued presence of cytokines, which exert pro-arrhythmic effect [16]. 


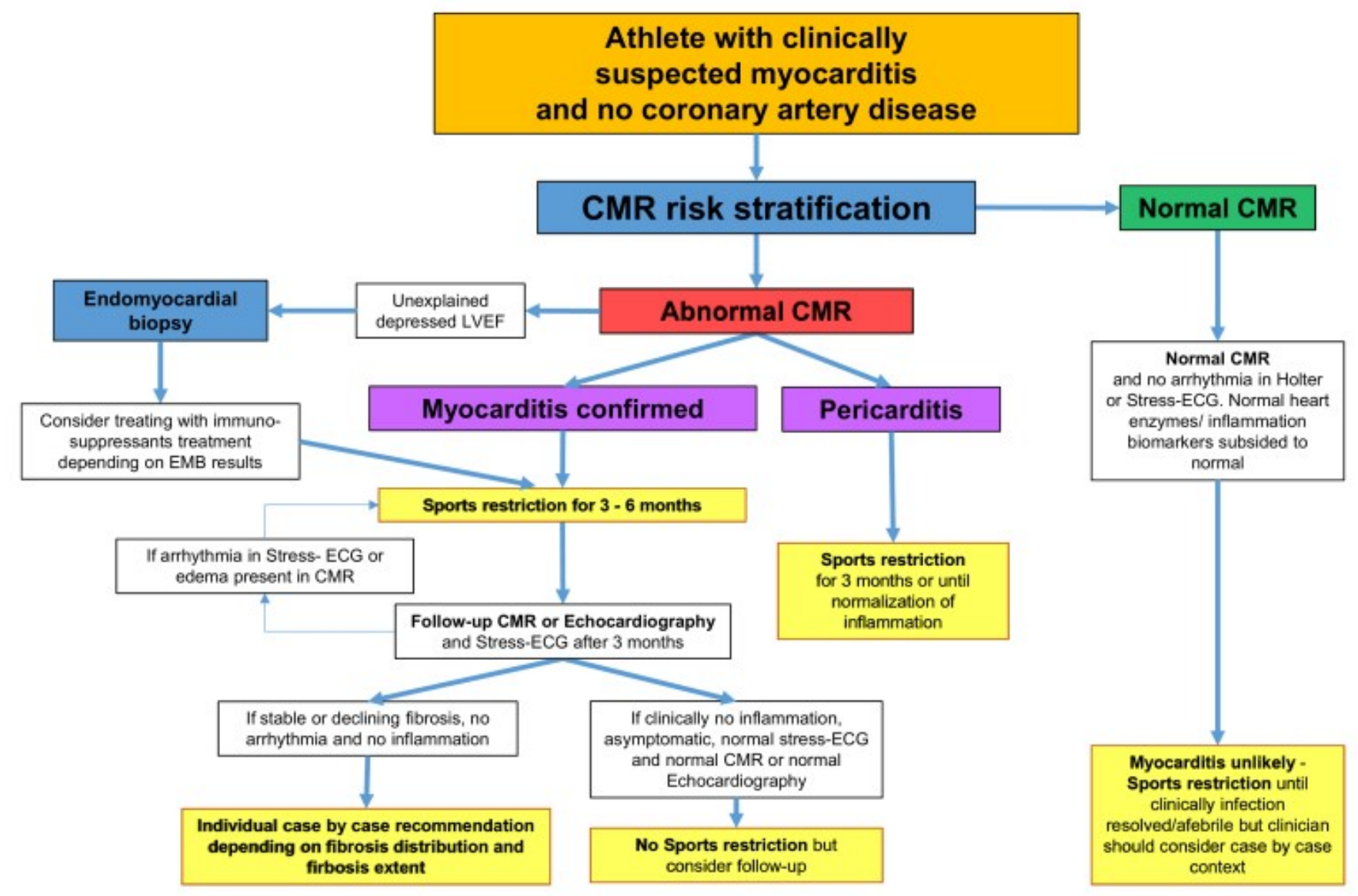

Figure 1: Diagnostic flow-chart and sports counselling of athletes with suspected myocarditis. Adapted from Eichhorn C. et al. JACC Cardiovasc Imaging, 2019.

\section{Clinical presentation and initial clinical work-up of athletes with suspected}

\section{myocarditis}

The typical clinical scenario is an athlete with atypical chest pain, aggravated when leaning forward with the upper body, history of a recent upper respiratory tract infection or gastrointestinal infection, elevated inflammatory and myocardial biomarkers, diffuse elevated ST-elevations and PQ depression in the electrocardiogram (ECG) and absence of coronary artery disease. However, this is only in the minority of the presentations the case. Myocarditis in athletes can present in a variety of ways. Beside chest pain as a leading symptom, patients can present with palpitations, performance drop or even be asymptomatic. Biomarkers, ECG and echocardiography might be abnormal, however, its sensitivity and specificity is low. As myocarditis may clinically resemble to an acute coronary event, it is important to rule out coronary artery disease (CAD) first in these patients. 


\section{Biomarkers and Electrocardiogram}

There are no specific biomarkers for myocarditis other than possible increased inflammation parameters C-reactive protein, leucocytes, elevated blood sedimentation rate and elevated myocardial biomarkers like troponin/creatin-kinase. Previous studies have shown that viral serology has a very low sensitivity and intermediate specificity with myocardial biopsy infiltrates confirming myocarditis [17]. Viral serology may be of use in ruling out hepatitis $\mathrm{C}$, human immunodeficiency virus, Lyme disease or rickettsia if there is a high suspicion. Myocarditis has been shown to be the second most common cause of high troponin levels in the young and middle-aged ( $<50$ years) [18], warranting further investigations once myocardial infarction has been ruled out. A dilemma is presented by the fact that endurance training can raise troponin levels as demonstrated by a meta-analysis totalling 1045 patients where troponin levels were raised above the cut-off in $83 \%$ of participants [19]. This means that repeat troponins in athletes are key in making the diagnosis of myocarditis as the raised troponin levels due to exercise resolve faster and are less prominent as well as monophasic in comparison to the troponin profiles found in myocarditis and myocardial infarction. Therefore, performing a troponin test in athletes suspected with myocarditis is important but it must be kept in mind that troponin levels do not sufficiently correlate with the level of oedema and scarring or recovery that can be observed on imaging modalities, such as cardiac magnetic resonance (CMR) imaging [20]. ECG abnormalities like PR depression, ST-elevations, T-wave inversion, Qwave presence, prolonged QRS or QTc may be present but are neither sensitive nor specific (see Figure 2) [21].

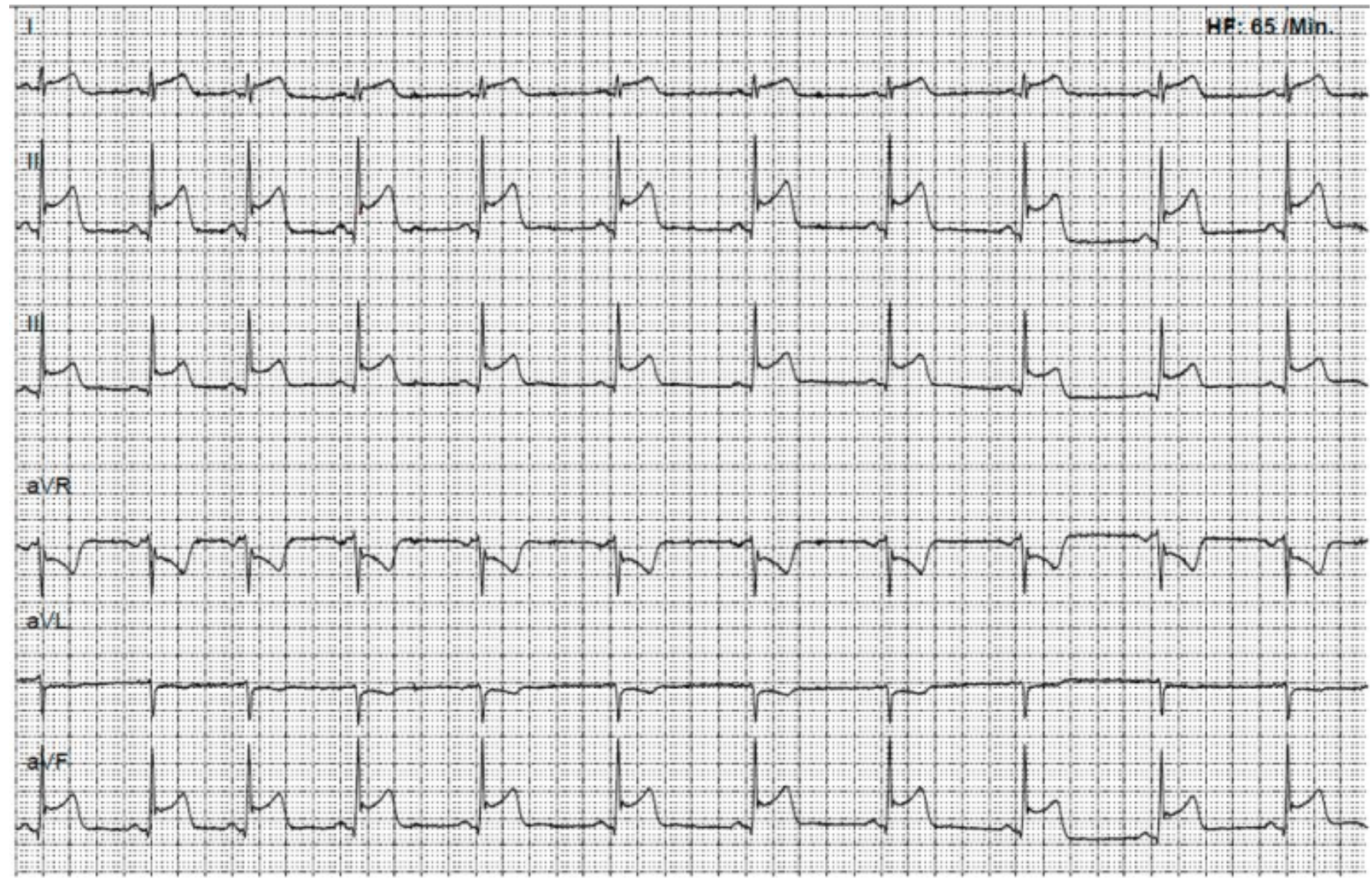


Figure 2: Case of an athlete with acute myocarditis. This is a case of a 17-year-old athlete with new onset of acute chest pain and pain in the left shoulder. He complained about a recent gastroenteritis three days ago. His body temperature was $39^{\circ} \mathrm{C}$ and C-reactive protein and Leucocytes were mildly elevated. Further, in laboratory tests, creatine kinase and troponin were elevated. Electrocardiogram showed diffuse ST-elevation over multiple leads. In order to rule out coronary ischemic event an invasive coronary angiogram was performed which was normal. Cardiac Magnetic resonance imaging was performed (see Figure 3).

\section{Echocardiography}

Pericardial effusion, local wall motion abnormalities or imaging consistent with dilated cardiomyopathy can be features of myocarditis. Fulminant myocarditis, a severe rapidly evolving form of myocarditis, shows severe impaired systolic LV function and is associated with a worse prognosis [22]. Echocardiography is an important tool to monitor LV function and wall motion abnormalities in myocarditis. It is crucial to differentiate myocarditis from an athlete's heart (Table 1), where the latter is a condition in which LV wall thickness and/or LV dimensions in athletes change depending on the degree of dynamic and static components of the type of exercise performed [23]. If there is any doubt about structural changes in an athlete's heart, further imaging, such as CMR imaging, is indicated [24].

\section{Endomyocardial Biopsy}

Endomyocardial biopsy (EMB) is not routinely performed in patients with suspected myocarditis, however, in cases with unclear depressed LV function or in life-threatening presentations, EMB helps to differentiate between infectious, autoimmune or idiopathic inflammation in myocarditis, which may guide further therapy [25,26]. While EMB has an outstanding specificity and low complication rates $(<1 \%)$ in experienced cardiac centres, sensitivity is low and there is a high chance of false negative results (sampling errors)

[25,27]. Whether the diagnostic value of EMB can be increased by using CMR imaging to guide the biopsy is currently unclear [25]. However, Baccouche et al. recently showed that using CMR and EMB in combination rather than individually identifies a greater proportion of patients with myocarditis [28]. Porcine pre-clinical in-vivo models suggest that real-time CMR-guided EMB may also improve sensitivity and specificity [29]. In regard to athletes, it is unknown whether EMB adds to risk stratification and sports abstinence counselling. 


\begin{tabular}{|c|c|c|}
\hline & $\begin{array}{l}\text { Differentiating Features } \\
\text { Athletes Heart }\end{array}$ & $\begin{array}{l}\text { Differentiating Features } \\
\text { Myocarditis }\end{array}$ \\
\hline Symptoms & Asymptomatic & Symptomatic \\
\hline ECG/Holter & $\begin{array}{l}\text { 1. Specific ECG changes such as early } \\
\text { repolarization/ST segment elevation. } \\
\text { T-wave inversion in V1-V3 in the } \\
\text { young, ST-elevation followed by } \\
\text { T-wave inversion V1-V4 in black } \\
\text { athletes. }\end{array}$ & $\begin{array}{l}\text { 1. Unspecific ECG changes. Possible } \\
P Q \text { depression, ST-elevation in mul- } \\
\text { tiple leads. }\end{array}$ \\
\hline Biomarkers/Inflammatory markers & $\begin{array}{l}\text { 1. Troponin elevation only mild and } \\
\text { normalizes quickly. May be present } \\
\text { in ultra-endurance athletes. } \\
\text { 2. Others: Brain natriuretic peptide } \\
\text { mildly elevated after ultra-endur- } \\
\text { ance exercise }\end{array}$ & $\begin{array}{l}\text { 1. Troponin elevation mild to high } \\
\text { 2. Others: Brain natriuretic peptide } \\
\text { elevation, Creatine-Kinase, Leuco- } \\
\text { cytosis, elevated C-reactive Protein, } \\
\text { elevated Erythrocyte sedimentation } \\
\text { rate }\end{array}$ \\
\hline
\end{tabular}

Table 1: Challenges in ECG, biomarkers and noninvasive imaging characteristics in patients with athlete's heart versus acute myocarditis.

\section{Cardiac Magnetic Resonance Imaging}

CMR is a rapidly evolving field and recent evidence suggests that current recommendations about the role of CMR in the management of patients with suspected myocarditis have to be updated. In 2009, the Consensus Criteria for Cardiovascular Magnetic Resonance in Myocardial Inflammation published the Lake Louise Criteria (LLC), which propose oedema by T2-weighted imaging and scar by late gadolinium enhancement (LGE) as diagnostic features for myocarditis (see Figure 3) [30]. CMR has not only diagnostic value

[31,32], but also a prognostic value as we recently could show. We analysed the prognostic value of CMR in a cohort that included 670 patients with suspected myocarditis. We could show that any LGE presence doubled the risk for major adverse cardiovascular outcomes (MACE), while septal and mid-wall LGE as well as patchy distribution of LGE were strong prognostic patterns of LGE [21]. In addition, increasing LGE extent resulted in a greater risk for outcome [33]. Further, T2 weighted imaging was also significantly associated with MACE [21]. Difficulties of CMR are that some biopsy-proven myocarditis patients do not show evidence of LGE and subsequently do not fulfil the LLC [25]. Additionally, it can be the case that the inflammatory process has become sufficiently diffuse across the myocardium to such an extent that T2 differences are not identifiable, especially if there is concurrent skeletal muscle inflammation in a reference region [34,35]. Novel T1 and T2 mapping sequences have been shown to provide higher sensitivity and specificity values than the traditional LLC criteria (see Figure 3)

[36,37]. T1 and T2 mapping values essentially describe magnetic properties on a pixel-by-pixel basis that are determined by intrinsic characteristics of the tissue, its environment and the measurement parameters, culminating in a global T1 or T2 value. The rationale behind T1 mapping values is that these are able to detect myocarditis throughout multiple stages of the disease course since they are affected by both oedema and extracellular expansion. T2 mapping can detect tissue free water content which is raised in the acute phase and normalizes over time - this can be important in differentiating myocarditis from other noninflammatory cardiomyopathies $[38,39]$. Furthermore, Spieker et al. have shown that T2 
mapping can be used as a prognostic marker in patients with myocarditis [40]. Using T1 mapping values pre and post contrast agent administration and adjusting for the haematocrit value, extracellular volume fraction (ECV) can also be estimated [41]. We have recently shown that there is a significant association of elevated ECV values with MACE in patients with suspected myocarditis, even in those presenting without any LGE [42]. All in all, it has become evident that inflamed myocardial tissue shows higher T1, T2 and ECV values, which compares favourably to other tissue characterisation techniques [43]. The recent update of the LLC implemented current evidence on novel mapping techniques and conclude that CMR provides strong evidence for an active myocardial inflammation based on either one T2-based parameter (T2 mapping or T2 weighted imaging) or one T1-based parameter (T1 mapping, ECV or LGE), with the specificity being higher when one of both parameters is present (44).
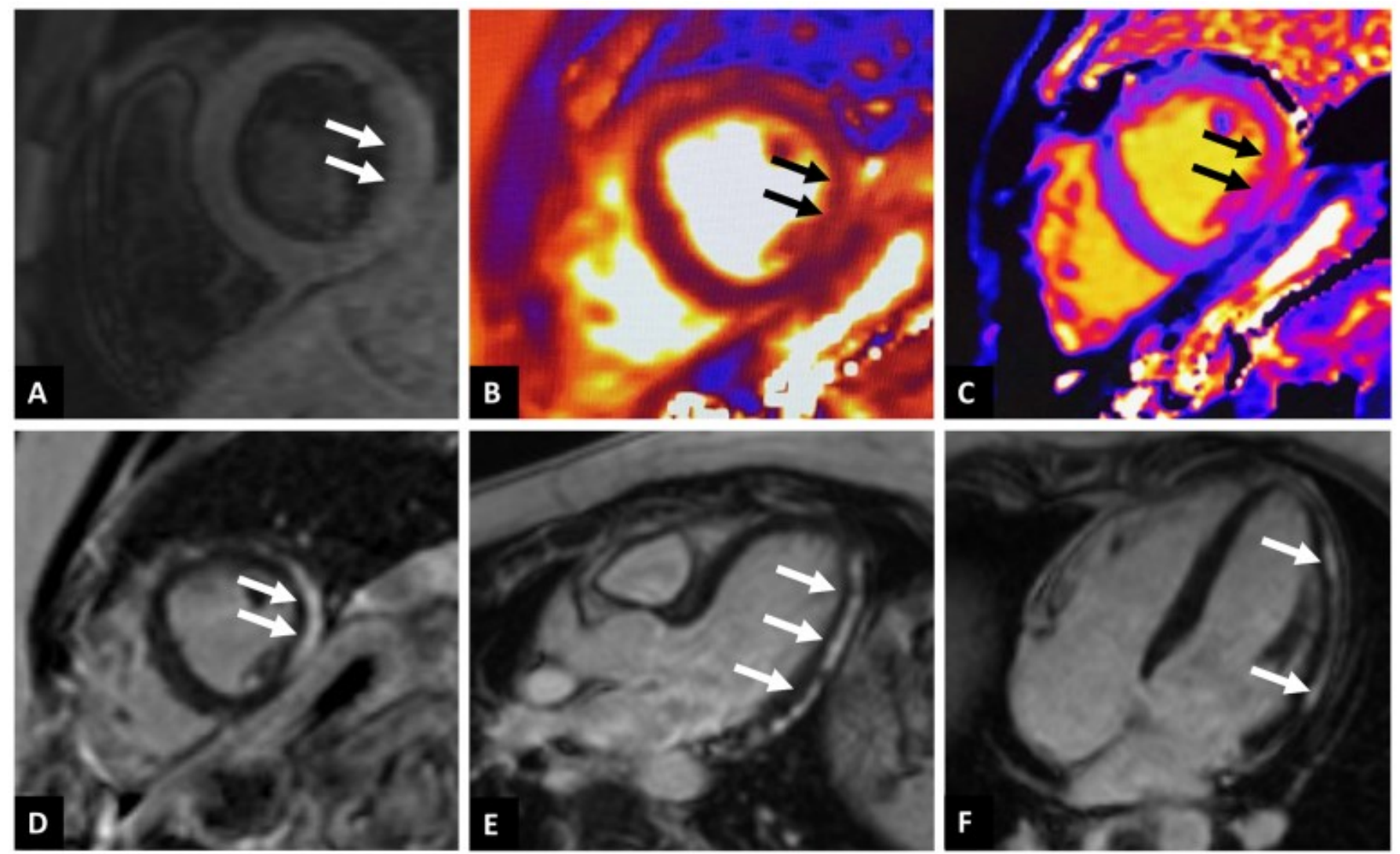

Figure 3: Cardiac Magnetic resonance imaging of an athlete with suspected myocarditis. The CMR of the athlete described in Figure 2 showed in panel A, the Turbo-Inversion Recovery- Magnitude (TIRM), i.e. a T2-weighted (water content) and fat supressed sequence of the midventricular short axis of the heart a hyperintensity signal of the epicardial left myocardial muscle inferolateral (white arrows). In the T2 mapping (panel B) sequence, in the same region, a prolonged T2 mapping time of 56ms could be measured consistent with myocardial oedema (black arrows). In the native T1 mapping (panel C), also inferolateral (midventricular) a prolonged T1 mapping time (1220ms) was measured (black arrows). Late Gadolinium enhancement is seen in a typical myocarditis pattern, i.e. subepicardial inferolateral from the basal to apical segments (panel D, short axis view, panel E, 3 chamber view and F 4 chamber view, white arrows). Left - and right ventricular function was normal without any regional wall motion abnormalities. The patient received non-steroidal anti-inflammatory drugs as was symptom free after discharge. He was counselled to restrict from competitive sports for 3 months and was scheduled for a follow-up CMR and maximal stress-ECG before 


\section{Therapy, Follow-up and Sports Restriction}

While roughly half of cases of suspected myocarditis resolve spontaneously within a month, there is a lack of evidence for optimal therapy in myocarditis patients with cardiac dysfunction. In haemodynamically stable patients, management of cardiac dysfunction should be in line with the latest heart failure guidelines [45] with appropriate step-up of care to intensive care units, invasive management and cardiac transplantation when patients become haemodynamically unstable. Arrhythmias and the use of implantable cardioverter defibrillator should also follow their respective guidelines. Current recommendation do not include immunomodulatory therapies, while immunosuppressive treatment, in the absence of active infection or other contraindications, is recommended in autoimmune forms of myocarditis.

In terms of sports restriction for athletes, it is paramount that athletes with acute myocarditis refrain from any competitive exercise, while there is currently no study that indicates that there is a safe level of exercise in the first months after a diagnosis of myocarditis. Current guidelines [9-11], including a recent recommendation by the European Association of Preventive Cardiology [12], recommend between 3 and 6 months of competitive sports restriction (Class IIb/Level C) for athletes diagnosed with myocarditis. Prior to returning to competitive exercise, athletes should undergo extensive testing, including echocardiography, Holter monitoring and exercise ECG. We believe that a follow-up CMR in athletes with diagnosed myocarditis is warranted at 3 to 6 months in order to risk stratify patients based on presence or absence of inflammation. These recommendations should not only apply to competitive athletes but also to recreational athletes as they form the group who most commonly suffer from SCD in myocarditis [46]. However, whether moderate exercise is safe when inflammation subsided to normal or whether different sports levels based on dynamic and static components may need different sport abstinence periods in athletes with myocarditis are unknown.

Furthermore, it needs to be evaluated how serial CMR scans and LGE involvement over time are associated with outcome.

\section{Conclusion}

In athletes with myocarditis, currently, 3-6 months sports restriction is recommended for the prevention of adverse cardiac events. CMR is the primary imaging tool for diagnosing myocarditis and may also play an important role in risk stratifying athletes with suspected myocarditis. Future research is needed to assess the role of novel noninvasive CMR tissue characterization techniques to better guide treatment and allow individual sports counseling in this clinical setting.

\section{Practical implications}

- The diagnosis of myocarditis in athletes is challenging

- Troponin levels are typically raised in myocarditis but athletic activity can also mildly increase troponin levels

- CMR is the primary tool to diagnose myocarditis and may further help to risk stratify athletes

- Currently 3-6 months competitive sports abstinence is recommended 


\section{Acknowledgments, conflict of interest and funding}

All authors have no conflict of interest. Dr. Gräni received funding support from the Swiss Sports Medicine Society (SGSM), the SGSM Award 2015.

\section{First author}

Christian Eichhorn, MBBS, BSc

Unterassistent

Department of Cardiology

Swiss Cardiovascular Center

University Hospital Berne

Freiburgstrasse

CH-3010 Berne, Switzerland



\section{Corresponding author}

PD Dr. med. Christoph Gräni, PhD

Director of noninvasive cardiac imaging

(CMR/CT/Nuclear)

Department of Cardiology

Swiss Cardiovascular Center

University Hospital Berne

Freiburgstrasse

CH-3010 Berne, Switzerland

Tel: +4131 6324508

Email: christoph.graeni@insel.ch

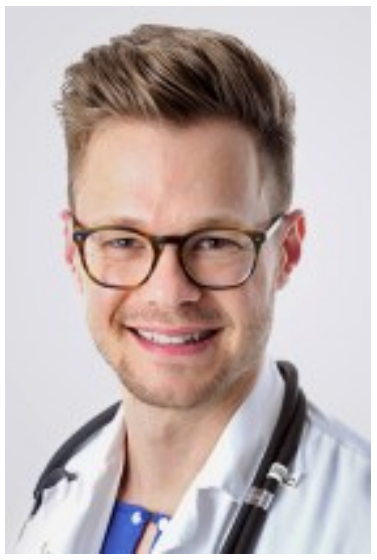

